\title{
Loop inflection-point inflation
}

\author{
Konstantinos Dimopoulos, ${ }^{1}$ Charlotte Owen, ${ }^{1}$ and Antonio Racioppi ${ }^{2}$ \\ ${ }^{1}$ Consortium for Fundamental Physics, Physics Department, \\ Lancaster University, Lancaster LA1 4YB, United Kingdom \\ ${ }^{2}$ National Institute of Chemical Physics and Biophysics, Rävala 10, 10143 Tallinn, Estonia
}

(Dated: July 5, 2018)

\begin{abstract}
A novel inflection-point inflation model is analysed. The model considers a massless scalar field, whose self-coupling's running is stabilised by a non-renormalisable operator. The running is controlled by a fermion loop. We find that successful inflation is possible for a natural value of the Yukawa coupling $y \simeq 4 \times 10^{-4}$. The necessary fine-tuning is only $\sim 10^{-6}$, which improves on the typical tuning of inflection-point inflation models, such as MSSM inflation. The model predicts a spectral index within the 1- $\sigma$ bound of the latest CMB observations, with a very small negative running, and negligible tensors $\left(r \sim 10^{-(9-10)}\right)$. These results are largely independent of the order of the stabilising non-renormalisable operator.
\end{abstract}

\section{INTRODUCTION}

Cosmic inflation is an organic part of concordance cosmology. With a single stroke inflation addresses the finetuning problems of the hot big bang; namely the horizon and flatness problems and also produces the primordial curvature perturbation, which seeds structure formation and is in excellent agreement with CMB observations [1]. According to the inflationary paradigm, the Universe undergoes inflation when dominated by the potential density of a scalar field, called the inflaton. However, the identity of the inflaton is as yet unknown.

The latest CMB observations suggest that the scalar potential of the inflaton features an inflationary plateau (e.g. see Ref [2]). Numerous mechanisms have been put forward to generate such a plateau, involving exotic constructions in the context of elaborate, beyond-the-standard-model theories, such as superstrings. One such example is inflectionpoint inflation, where the inflationary plateau is due to the interplay of opposing contributions in the scalar potential, which (almost) cancel each other out generating a step on the otherwise steep potential wall. The original model was called A-term inflation, because it employed the A-term of a supersymmetric theory [3, 4], or MSSM inflation, because it considered a flat direction in MSSM [5] as the inflaton. However, other models of inflection-point inflation have also been constructed $[6,7]$. Most of these also consider an elaborate setup in the context of supersymmetry, string theory or other extensions of the Standard Model.

However, an advantage of the idea of inflation is that it does not have to rely on exotic physics, in contrast to alternatives like the ekpyrotic scenario [8] or string gas cosmology [9]. Indeed, inflation may be realised simply within field theory in curved spacetime. It is also possible to achieve inflection-point inflation in this way. In this paper we explore such a possibility, where we exploit the loop corrections to the inflaton potential to generate the steplike plateau. This is similar to the works in Ref. [7]. However, in Ref. [7] the authors consider a rather complicated running of the inflaton self-coupling, where many particles are contributing to it. We consider a simpler setup.

In previous works care was taken so that loop corrections do not spoil the stability of the potential [10]. In contrast, here we consider a model in which the Coleman-Weinberg potential is unstable. Stability is recovered by introducing a Planck-suppressed effective operator.

We use natural units where $c=\hbar=1$ and $8 \pi G=m_{P}^{-2}$, with $m_{P}=2.43 \times 10^{18} \mathrm{GeV}$ being the reduced Planck mass.

\section{COLEMAN-WEINBERG POTENTIAL}

The general expression for the 1-loop potential is given by the Coleman-Weinberg $(\mathrm{CW})$ result [11]

$$
V_{\mathrm{eff}}=V+\sum_{i=1}^{n} \frac{g_{i} M_{i}^{4}(\phi)}{64 \pi^{2}} \ln \left(\frac{M_{i}^{2}(\phi)}{\mu^{2}}\right)
$$

where $V$ is the tree-level potential, $\mu$ is the renormalisation scale and $M_{i}$ and $g_{i}$ are, respectively, the field dependent tree level mass and the number of intrinsic degrees of freedom of the particle- $i$ coupled with $\phi$. We assume a quartic tree-level potential for the inflaton field

$$
V=\lambda \phi^{4},
$$

and that the dominant contribution in Eq. (1) is given by the Yukawa coupling $y$ between $\phi$ and a Weyl fermion ${ }^{1}$. Therefore we can approximate Eq. (1) with

$$
V_{\mathrm{eff}}(\phi)=\left[\lambda-\beta \ln \left(\frac{y^{2} \phi^{2}}{\mu^{2}}\right)\right] \phi^{4},
$$

where we used Eq. (2) and $\beta=y^{4} / 32 \pi^{2}$. We can improve the potential by inserting the running expression for

\footnotetext{
1 A similar computation can be performed also in the case of more fermionic degrees of freedom. However, since here we are not discussing the details of the fermion sector phenomenology, but just its contribution to the effective potential, we limit ourselves to the minimal setup.
} 
$\lambda$. Since we assumed that the Yukawa coupling $y$ is the dominant contribution, a good approximation ${ }^{2}$ for the RGE solution of $\lambda$ is

$$
\lambda(\mu)=\lambda(M)-2 \beta \log \left(\frac{\mu}{M}\right),
$$

where $M$ is the scale at which we impose the boundary condition on the running of $\lambda$. Since we are interested in studying a configuration in which the $\mathrm{CW}$ potential is unstable, it is natural to pick ${ }^{3} \lambda(M)=0$. Using this and inserting Eq. (4) into Eq. (3) we get

$$
V_{\mathrm{eff}}(\phi)=-\beta \ln \left(\frac{y^{2} \phi^{2}}{M^{2}}\right) \phi^{4} .
$$

\section{INFLATION MODEL WITH INFLECTION POINT}

The potential in Eq. (5) is not stable because it is unbounded from below. We assume that stability is ensured by the intervention of a non-renormalisable Plancksuppressed effective operator. Therefore let us consider the following inflaton potential

$$
V=-\beta \ln \left(\frac{y^{2} \phi^{2}}{M^{2}}\right) \phi^{4}+\lambda_{n} \frac{\phi^{2 n+4}}{m_{P}^{2 n}},
$$

where the first term is the 1-loop effective potential obtained in Eq. (5) and the second term is an effective nonrenormalisable operator, with $\lambda_{n} \ll 1$ and $n \geq 1$. We consider only the dominant non-renormalisable term, of order $n$.

For the moment we choose $n=1$ but later on we consider higher values of $n$. For simplicity, we study the model where

$$
\frac{y^{2}}{M^{2}}=\frac{1}{m_{P}^{2}}
$$

If $y<1$ (required for pertubativity), it is possible to realise such a condition with sub-Planckian $M$.

A priori, $M$ and $y$ can take whatever possible value. However it is possible to reduce the parameters space, identifying a preferred region which is essentially described by Eq. (7). For example, assuming that our inflaton is not the Higgs boson of the SM, it is reasonable to expect new physics to happen around the scale of grand unification (GUT-scale). Therefore it is reasonable to consider $M \sim 10^{15-16} \mathrm{GeV}$. In addition to that, the Yukawa

2 There is also a RGE for $y$ to be solved. In a minimal setup in which the Weyl fermion is only coupled to $\phi$, the beta function for such a coupling would behave as $\beta_{y} \approx y^{3}$. If $y \ll 1$, then the running of $y$ becomes negligible and $y$ can be safely treated as a constant.

3 The choice is just a convenient parametrization. Even if we would assume $\lambda(M) \neq 0$, we can always find a new scale $M^{*}=M \exp \left(\frac{\lambda(M)}{2 \beta}\right)$ at which $\lambda\left(M^{*}\right)=0$. Therefore the computations would then proceed in the same way from Eq. (5) with simply $M^{*}$ in place of $M$. coupling, $y$, generating the loop correction must be small enough to preserve perturbativity, but on the other side, also big enough to give rise to relevant corrections. Therefore a reasonable range for $y$ is ${ }^{4}$ around $10^{-(2-3)}$. Combining the two expected regions for $M$ and $y$, we get that $y / M$ is around $1 / m_{P}$, therefore for the first analysis, in which we present a new idea for inflection point models, it is enough to study the model implementing Eq. (7). We will consider a broader range of $M$ and $y$ values in a future article.

Noting that the slow-roll formalism is independent of the potential normalisation, we reparametrise the potential as

$$
V=\beta\left[-\ln \left(\frac{\phi^{2}}{m_{P}^{2}}\right) \phi^{4}+\alpha \frac{\phi^{6}}{m_{P}^{2}}\right],
$$

where $\alpha=\lambda_{1} / \beta$. Such a potential has a flat inflection point at

$$
\phi_{f}=e^{1 / 4} m_{P} \quad \text { and } \quad \alpha_{f} \equiv \frac{2}{3 \sqrt{e}} .
$$

To study the inflationary predictions for values of $\alpha$ around $\alpha_{f}$, we parametrise:

$$
\alpha=(1+\delta) \alpha_{f}
$$

and use $\delta$ as a free parameter. Varying $\delta$ allows us to find the range of allowed slopes of the plateau around the flat inflection point. Increasing $\delta$ increases the slope of the plateau. Decreasing $\delta$ to negative values introduces a local maximum.

There are two aspects to consider when constraining $\delta$. First, by contrasting the computed inflationary observables with the observations. Second, by ensuring that the necessary remaining e-folds of inflation since the cosmological scales exited the horizon, $N_{*}$, is not greater than the total e-folds of inflation, $N_{\text {tot }}$. When the parameter space for $\delta$ is established we calculate predictions for the inflationary observables, namely the spectral index of the scalar curvature perturbations, $n_{s}$, its running, $n_{s}^{\prime} \equiv \frac{d n_{s}}{d \ln k}$ and the tensor-to-scalar ratio, $r$.

\section{Computing $N_{*}$}

First we must make clear the distinction between $N_{\text {tot }}$ and $N_{*}$. $N_{\text {tot }}$ depends mainly on the initial conditions of the inflaton. We set the beginning of inflation to be determined by $\epsilon=1$, where $\epsilon=-\dot{H} / H^{2}$ is the usual slow-roll parameter. For the e-folds of observable inflation $N_{*}$, typically the reheating temperature has a large impact. However, our model does not need an in-depth investigation

\footnotetext{
${ }^{4}$ Indeed, we find $y=4 \times 10^{-4}$ (see conclusions), which is not that far from the expected range.
} 
into reheating since in this model, after inflation, the field oscillates in a quartic minimum because of Eq. (2) and also

$$
\lim _{\phi \rightarrow 0}\left[-\beta \ln \left(\frac{\phi^{2}}{m_{P}^{2}}\right) \phi^{4}\right]=\frac{1}{2} \beta \phi^{4} .
$$

The average density of a scalar field coherently oscillating in a quartic potential scales as $\rho_{\phi} \propto a^{-4}$ [12], just as the density of a radiation dominated Universe. Hence, there is little distinction in the expansion between inflaton oscillations and radiation domination after reheating, which means that $N_{*}$ is independent of the inflaton decay rate.

In this case we have

$$
N_{*}=62.8-\ln \left(\frac{k}{a_{0} H_{0}}\right)+\frac{1}{3} \ln \left(\frac{g_{*}}{106.75}\right)+\frac{1}{3} \ln \left(\frac{V_{\text {end }}^{1 / 4}}{10^{16} \mathrm{GeV}}\right)
$$

where $k=0.05 \mathrm{Mpc}^{-1}$ is the pivot scale, $\left(a_{0} H_{0}\right)^{-1}$ is the comoving Hubble radius today, $g_{*}$ is the effective number of relativistic degrees of freedom and $V_{\text {end }} \equiv V\left(\phi_{\text {end }}\right)$, with 'end' denoting the end of inflation. This simplifies when we take $g_{*}=106.75$, corresponding to the standard model at high energies. Inputting the values of $k$ and $a_{0} H_{0}$ as well, gives

$$
N_{*}=57.4+\frac{1}{3} \ln \left(\frac{V_{\mathrm{end}}^{1 / 4}}{10^{16} \mathrm{GeV}}\right) .
$$

\section{Limits of $\delta$}

$N_{\text {tot }}$ can be calculated by integrating between the two values of $\phi$ that result in $\epsilon=1$, marking the beginning and end points of slow roll inflation. If $N_{*} \simeq N_{\text {tot }}$ we may need to investigate the initial conditions of $\phi$ to assess whether or not slow-roll does start at $\epsilon=1$. This will depend on whether or not the inflaton is kinetically dominated when it reaches the plateau. Ensuring $N_{\text {tot }}>N_{*}$ imposes a maximum value for $\delta$ :

$$
\delta<10^{-5.16}
$$

\section{Inflationary Observables}

The spectral index and tensor-to-scalar ratio for this model are calculated for varying positive $\delta$ values and the parameter space satisfying the Planck results is presented in Table I, along with the values of $N_{*}, N_{\text {tot }}$ and the running of the spectral index. Using the Planck $2-\sigma$ constraint of $n_{s}=0.968 \pm 0.010[1]$, provides limits on $\delta$ :

$$
10^{-6.06} \leq \delta \leq 10^{-5.86} .
$$

It is clear that, for the region where the spectral index and tensor-to-scalar ratio values match observations, $\delta$ is within the constraint of Eq. (14) such that $N_{*}<N_{\text {tot }}$ and we do not need to worry about initial conditions.

The model's predictions for the inflationary observables are shown in Table I and Fig. 1.

\begin{tabular}{|c|c|c|c|c|c|}
\hline$\delta$ & $N_{*}$ & $N_{\text {tot }}$ & $n_{s}$ & $r / 10^{-9}$ & $n_{s}^{\prime} / 10^{-6}$ \\
\hline $10^{-5.80}$ & 56.21 & 120.99 & 0.987 & 7.24 & -3.72 \\
\hline $10^{-5.85}$ & 56.19 & 128.18 & 0.980 & 6.07 & -3.11 \\
\hline $10^{-5.90}$ & 56.18 & 135.79 & 0.973 & 5.21 & -2.67 \\
\hline $10^{-5.95}$ & 56.17 & 143.84 & 0.968 & 4.55 & -2.33 \\
\hline $10^{-6.00}$ & 56.16 & 152.38 & 0.963 & 4.04 & -2.07 \\
\hline $10^{-6.05}$ & 56.15 & 161.23 & 0.959 & 3.64 & -1.86 \\
\hline $10^{-6.10}$ & 56.14 & 171.00 & 0.955 & 3.32 & -1.70 \\
\hline
\end{tabular}

TABLE I: $\delta$ values producing $n_{s}$ within the Planck $2-\sigma$ bounds.

\section{NEGATIVE $\delta$ VALUES AND QUANTUM TUNNELLING}

When $\delta$ is negative, the potential develops a local minimum and maximum in place of a flat plateau. When the inflaton field tunnels through the local maximum, it may be in a position to slow-roll on the other side of the peak, hopefully for enough e-folds to generate $n_{s}$ and $r$ in accordance with observations. We calculated the number of slow-roll e-folds from the exit point of the quantum tunnelling and found that they are enough only when $\delta \geq-10^{-8}$ and in all cases $N_{*}=56.08$. Even though this is a similar e-folding number to our results in the positive delta case, because the

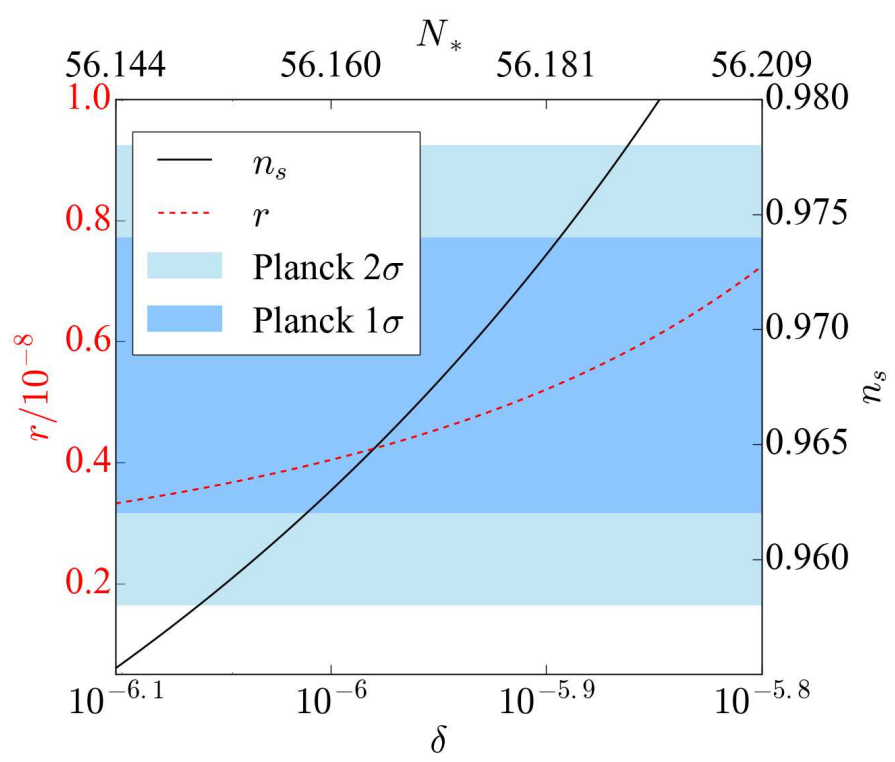

FIG. 1: Values of $\delta$ for which $n_{s}$ (solid black line) and $r$ (dashed red line) fall within the Planck bounds for $n_{s}$ depicted with the shaded horizontal bands (light: $2-\sigma$ and darkened: $1-\sigma)$. The top axis also shows the corresponding $N_{*}$ values for each $\delta$ value. (CMB only provides a weak bound on $r<0.1[1]$ ) 
delta value is constrained to be a lot smaller it only results in $n_{s}=0.928$ which is unacceptable because the spectrum is too red.

\section{HIGHER-ORDER NON-RENORMALISABLE TERM}

Let us now consider higher values of $n$; the order of the non-renormalisable operator in Eq. (6). It is straightforward to check that our findings in the $n=1$ case are largely unchanged. As shown in Tables III, IV and V, for $n=2,3,4$, we find $n_{s}$ within the 2- $\sigma$ Planck bounds only when $10^{-6.2}<\delta<10^{-5.9}$. We also find $n_{s}^{\prime} \sim-10^{-6}$, $N_{*} \approx 56, N_{\text {tot }}>2 N_{*}$ and $r \sim 10^{-(9-10)}$. Our results show $r \simeq 0$, with very small differences for varying $n$, and changes in $n_{s}$ for varying $n$ are at the $10^{-3}$ level. It should not be a surprise that our results are robust and largely independent of $n$, as is clearly shown in Fig. 2. By the time the cosmological scales leave the horizon the field has rolled passed the inflection point and the scalar potential is dominated by the CW-term in Eq. (5), which is $n$ independent.

\begin{tabular}{|c|c|c|c|c|}
\hline$n$ & 1 & 2 & 3 & 4 \\
\hline$\left(\phi_{f} / m_{P}\right)^{2 n}$ & 1.65 & 1.00 & 0.61 & 0.37 \\
\hline
\end{tabular}

TABLE II: Values of $\left(\phi_{f} / m_{P}\right)^{2 n}$ for $n \geq 1$.

The value of the field at the inflection point $\phi_{f}$ reduces somewhat for larger $n$. Indeed, it is easy to show that the generalisation of Eq. (9) for arbitrary $n$ is

$$
\phi_{f}=e^{\frac{1}{2 n}\left(1-\frac{n}{2}\right)} m_{P} \quad \text { and } \quad \alpha_{f} \equiv \frac{2 e^{\frac{n}{2}-1}}{n(n+2)} .
$$

The above suggest that $\left(\phi_{f} / m_{P}\right)^{2 n}=e^{1-\frac{n}{2}}$, which means that $\left(\phi_{f} / m_{P}\right)^{2 n} \lesssim 0.1$ for $n \geq 4$ (see Table II). This implies that, because $\phi<\phi_{f}$ when the cosmological scales exit the horizon $\left(N_{*}<N_{\text {tot }} / 2\right)$, we would expect high-order non-renormalisable terms to be suppressed when $n>4$. Thus, it is unlikely that the dominant, stabilising, nonrenormalisable operator would correspond to $n>4$.

\begin{tabular}{|c|c|c|c|c|c|}
\hline$\delta$ & $N_{*}$ & $N_{\text {tot }}$ & $n_{s}$ & $r / 10^{-10}$ & $n_{s}^{\prime} / 10^{-6}$ \\
\hline $10^{-5.9}$ & 56.04 & 123.60 & 0.984 & 8.51 & -3.48 \\
\hline $10^{-6.0}$ & 56.01 & 138.70 & 0.971 & 6.2 & -2.54 \\
\hline $10^{-6.1}$ & 55.99 & 155.64 & 0.961 & 4.88 & -1.99 \\
\hline $10^{-6.2}$ & 55.97 & 174.65 & 0.954 & 4.05 & -1.65 \\
\hline
\end{tabular}

TABLE III: Results for $\phi^{8}$

\begin{tabular}{|c|c|c|c|c|c|}
\hline$\delta$ & $N_{*}$ & $N_{\text {tot }}$ & $n_{s}$ & $r / 10^{-10}$ & $n_{s}^{\prime} / 10^{-6}$ \\
\hline $10^{-5.9}$ & 55.95 & 126.21 & 0.981 & 3.1 & -3.27 \\
\hline $10^{-6.0}$ & 55.93 & 141.81 & 0.969 & 2.30 & -2.42 \\
\hline $10^{-6.1}$ & 55.91 & 159.39 & 0.960 & 1.82 & -1.92 \\
\hline $10^{-6.2}$ & 55.90 & 179.23 & 0.953 & 1.52 & -1.60 \\
\hline
\end{tabular}

TABLE IV: Results for $\phi^{10}$

\begin{tabular}{|c|c|c|c|c|c|}
\hline$\delta$ & $N_{*}$ & $N_{\text {tot }}$ & $n_{s}$ & $r / 10^{-10}$ & $n_{s}^{\prime} / 10^{-6}$ \\
\hline $10^{-5.9}$ & 55.91 & 125.30 & 0.982 & 1.72 & -3.35 \\
\hline $10^{-6.0}$ & 55.88 & 142.71 & 0.970 & 1.29 & -2.51 \\
\hline $10^{-6.1}$ & 55.86 & 158.58 & 0.961 & 1.03 & -2.00 \\
\hline $10^{-6.2}$ & 55.85 & 172.96 & 0.954 & 0.87 & -1.68 \\
\hline
\end{tabular}

TABLE V: Results for $\phi^{12}$

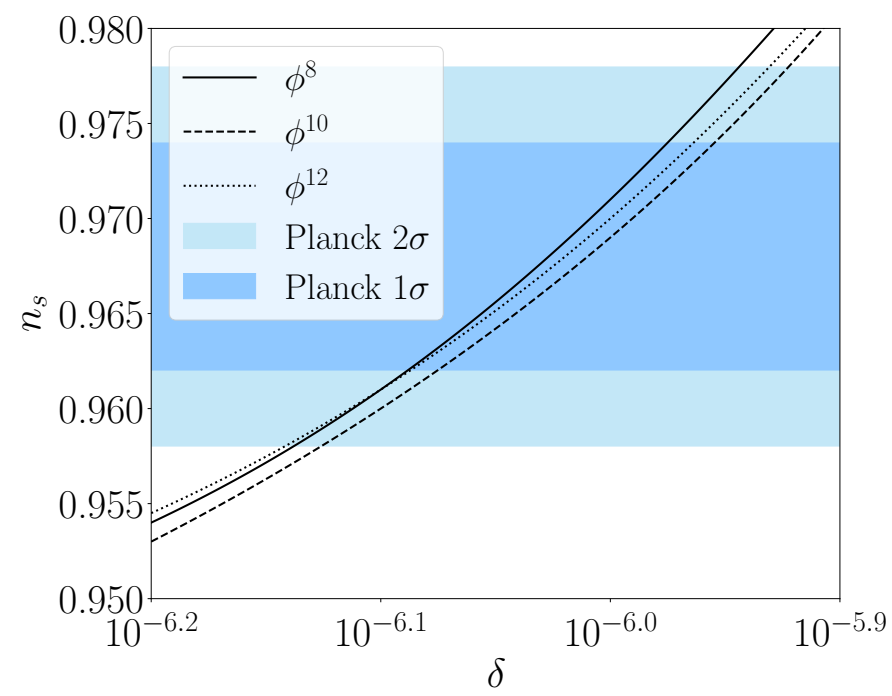

FIG. 2: Values of $\delta$ for which $n_{s}$ falls within the Planck bounds depicted with the shaded horizontal bands (light: $2-\sigma$ and darkened: $1-\sigma$ ) for varying orders of the non-renormalisable term, $n$. 


\section{INFLATIONARY SCALE AND FINE-TUNING}

We determine the inflationary energy scale via the COBE constraint:

$$
V^{1 / 4}=0.013 r^{1 / 4} m_{P} .
$$

As shown in Table I and Tables III, IV and V, for $\delta \sim 10^{-6}$, we have $r \sim 10^{-(9-10)}$. Thus, the above suggests that $V^{1 / 4} \sim 10^{14} \mathrm{GeV}$. Now, from Eq. (8), we have ${ }^{5}$ $V^{1 / 4} \sim \beta^{1 / 4} \phi$. Using the fact that $\phi \sim \phi_{f} \sim m_{P}$ we obtain $\beta \sim 10^{-16}$. Because $\beta=y^{4} / 32 \pi^{2}$, we find $y \simeq 4 \times 10^{-4}$, which is a very reasonable value for a Yukawa coupling and in agreement with the assumption $y \ll 1$ (see Eq. (4) and footnote 2). Through Eq. (7), we then determine $M=2 \sqrt{\pi}(2 \beta)^{1 / 4} m_{P} \simeq 10^{15} \mathrm{GeV}$; near the grand unification scale and sub-Planckian as expected.

Inflection-point inflation involves fine-tuning to attain the necessary inflationary plateau. In loop inflectionpoint inflation the tuning ${ }^{6}$ is $\delta \sim 10^{-6}$. This is exponentially better than the tuning corresponding to the horizon and flatness problems, resolution of which is one of the main motivations of inflation. For example, at the scale $V^{1 / 4} \sim 10^{14} \mathrm{GeV}$, the deviation from flatness needs to be $|\Omega-1| \lesssim 10^{-40}$. Note also, that $\delta \sim 10^{-6}$ is much better than the level of tuning required in A-term/MSSM inflation [4].

It is important to note here that the assumption of slowroll is not always justified in inflection-point inflation models. This is because the potential near the inflection-point is so flat that the system may depart from slow-roll and temporarily engage into so-called ultra-slow-roll (USR) inflation $[13]^{7}$. This can have profound implications on the calculation of inflationary observables and may invalidate our findings (as well as those of most of the inflectionpoint literature). However, this danger can by averted if we assume that the inflaton lies initially near the inflection point with small enough kinetic density. In Ref. [14] it is shown that, when the original kinetic density satisfies the bound $\rho_{\text {kin }} \leq\left(V^{\prime} m_{P}\right)^{2} / 6 V$ at the inflection point, then slow-roll inflation begins immediately and all our findings are reliable. In our model $V \sim \beta m_{P}^{4}$ and it can be easily shown that $V^{\prime}\left(\phi_{f}\right) \sim \beta \delta m_{P}^{3}$. This means that, if the inflaton starts near $\phi_{f}$ with kinetic density $\rho_{\text {kin }} \lesssim \beta \delta^{2} m_{P}^{4}$ then slow-roll inflation begins immediately and our findings are fine. Putting in the numbers, we find $\rho_{\text {kin }}^{1 / 4} \lesssim 10^{11} \mathrm{GeV}$,

\footnotetext{
5 Strictly speaking, Eq. (8) considers $n=1$. However, observable inflation occurs after the inflaton field crosses the inflection point $\phi_{f}$, which means that the $\mathrm{CW}$ term dominates over the non-renormalisable term in Eq. (8). Thus, the order of the nonrenormalisable term is not relevant here and $V^{1 / 4} \sim \beta^{1 / 4} \phi$ for $n>1$ too.

6 This does not take into account the tuning required to satisfy Eq. (7). However such tuning is rather small since Eq. (7) is satisfied for quite natural values of the parameters.

7 We would like to thank C. Germani for pointing this out.
}

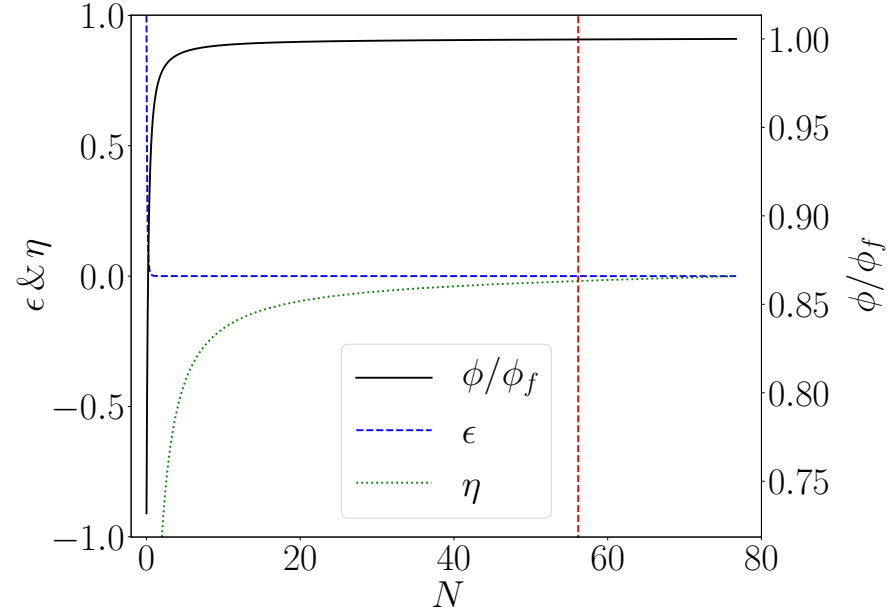

FIG. 3: The values of the inflaton field $\phi$ (solid black line) and the slow-roll parameters $\epsilon$ (dashed blue line) and $\eta$ (dotted green line) with respect to the remaining e-folds of inflation $N$ are shown. The system progresses from right to left and inflation ends when $N=0$. The vertical (dashed red) line denotes $N_{*}$, which corresponds to the time when the cosmological scales exit the horizon during inflation. The inflaton is taken to roll from the inflection point at $\phi_{f}$ with negligible initial kinetic density, such that the slow-roll attractor is immediately assumed. As shown, $\epsilon$ is kept exponentially small during inflation. Inflation ends when $\epsilon=1$, with $|\eta|$ becoming large in the last e-fold of inflation, corresponding to a substantial variation of $\epsilon$, that ends inflation.

i.e. a factor of $10^{3}$ smaller than the energy scale of inflation. In Fig. 3 we track the evolution of the inflaton field and slow-roll parameters during inflation to demonstrate the avoidance of USR for negligible initial kinetic energy densities at the inflection point. Note that the issue of the initial conditions of inflation is academic because of the no-hair theorem, which demonstrates that memory of the initial conditions is lost once the inflationary attractor is attained.

\section{REHEATING}

A theory with $V \sim \beta \phi^{4}$, when $V^{\prime \prime}>H^{2}$ leads to coherent oscillations in a quartic potential, whose density scales as radiation $\rho \propto a^{-4}[15]$. This means that the amplitude of the oscillations decreases as $\phi \propto 1 / a$. These oscillations correspond to particles of mass $m \sim \sqrt{\beta} \phi \propto 1 / a$, which is redshifted similarly to radiation particles [16].

The decay rate of the inflaton particles to the Weyl fermions they couple to is $\Gamma=y^{2} m / 8 \pi$. This means that, after inflation and during the oscillations, $\Gamma / H \propto a$, since $H \propto a^{-2}$. Thus, because $\Gamma / H$ increases in time, there will be a moment when $\Gamma \sim H$ and the decay becomes efficient and it leads to reheating. Here we assume that the Weyl 
fermions produced by the inflaton decay are coupled to SM particles such that, once formed, they promptly decay into the radiation bath of the hot big bang.

Therefore, reheating occurs when

$$
\frac{a_{\mathrm{reh}}}{a_{\text {end }}} \sim \frac{H_{\text {end }}}{\Gamma_{\text {end }}} \sim \frac{1}{\sqrt{\beta}} \frac{8 \pi}{y^{2}} \frac{H_{\text {end }}}{\phi_{\text {end }}},
$$

where 'reh' denotes the moment of reheating. Using that the amplitude of the oscillations decreases as $\phi \propto 1 / a$, the above gives

$$
\phi_{\mathrm{reh}} \sim \frac{y^{2} \sqrt{\beta}}{8 \pi} \frac{\phi_{\mathrm{end}}^{2}}{H_{\mathrm{end}}} .
$$

For the density of the oscillating condensate we have

$$
\rho_{\text {reh }}=\rho_{\text {end }}\left(\frac{a_{\text {end }}}{a_{\text {reh }}}\right)^{4} \sim 9 \beta\left(\frac{y^{2}}{8 \pi}\right)^{4} m_{P}^{4},
$$

where we used $\rho_{\text {end }} \sim V_{\text {end }} \sim \beta \phi_{\text {end }}^{4}$ and $H_{\text {end }}^{2}=\rho_{\text {end }} / 3 m_{P}^{2}$.

For the radiation bath we have $\rho_{\text {reh }}=\left(\pi^{2} / 30\right) g_{*} T_{\text {reh }}^{4}$, where $g_{*}$ is the effective relativistic degrees of freedom and $T_{\text {reh }}$ is the reheating temperature. Using this, the above equation suggests

$$
T_{\mathrm{reh}} \sim\left(\frac{270}{\pi^{2} g_{*}}\right)^{1 / 4} \frac{y^{2} \beta^{1 / 4}}{8 \pi} m_{P} \sim 0.03 y^{2} \beta^{1 / 4} m_{P},
$$

where we considered that $g_{*}=\mathcal{O}(100) .{ }^{8}$ Putting the numbers we obtained: $y \simeq 4 \times 10^{-4}$ and $\beta \sim 10^{-16}$, we get $T_{\text {reh }} \sim 10^{6} \mathrm{GeV}$, which is comfortably higher than the temperature at BBN $(\sim 1 \mathrm{MeV})$ but low enough to avoid the generation of dangerous relics (e.g. gravitinos).

Now suppose that there is also a quadratic term in the scalar potential such that the inflaton has a bare mass $m_{0}$ and $V \sim m_{0}^{2} \phi^{2}+\beta \phi^{4}$. In order not to influence inflation, the quadratic term must remain negligible during inflation. This means $m_{0}^{2}<\beta \phi^{2}$. Using $\beta \sim 10^{-16}$ and $\phi \sim \phi_{f} \sim m_{P}$, we find the bound $m_{0}<10^{10} \mathrm{GeV}$.

In order not to influence reheating the bound on $m_{0}$ is much more stringent, because we need the quadratic term in the potential to remain subdominant until the decay of the inflaton condensate, that is we need $m_{0}^{2}<\beta \phi_{\text {reh }}^{2}$. In view of Eq. (19), we get

$$
m_{0}<\sqrt{3 \beta} \frac{y^{2}}{8 \pi} m_{P},
$$

where we also considered that $H_{\text {end }}^{2} \simeq V_{\text {end }} / 3 m_{P}^{2}$ and $V_{\text {end }} \sim \beta \phi_{\text {end }}^{4}$. Putting the numbers in, we obtain $m_{0}<300 \mathrm{GeV}$ or so. This is a bit tight but it also means that if $m_{0} \sim 1 \mathrm{TeV}$, the influence on the value of $N_{*}$ would be of the order $\Delta N_{*} \simeq \frac{1}{6} \ln \left(m_{0}^{2}-\beta \phi_{\text {reh }}^{2}\right)<1$, which would have minimal impact on our results, while a $\mathrm{TeV}$-scale scalar particle might be observable in the LHC in the near future.

\footnotetext{
8 This is Eq. (108) of Ref. [16].
}

\section{CONCLUSIONS}

To conclude, we have studied a simple but elegant inflation model, where the inflationary plateau is generated through the running of the self-coupling of a massless scalar field, stabilised by a non-renormalisable operator. We have found that the model accounts for observations with mild tuning of the order $\sim 10^{-6}$ and a natural value of the Yukawa coupling $y \simeq 4 \times 10^{-4}$. In particular, the model can result in the spectral index of the scalar curvature perturbation within the 1- $\sigma$ bound of the latest $\mathrm{CMB}$ observations, while producing negligible tensors $\left(r \sim 10^{-(9-10)}\right)$. The inflationary energy scale is $V \sim 10^{14} \mathrm{GeV}$; much higher that A-term/MSSM inflation (hence, the tuning is less). We also studied perturbative reheating in our model and obtained a reasonable reheating temperature $T_{\text {reh }} \sim 10^{6} \mathrm{GeV}$. Non-perturbative effects might enhance the efficiency of reheating. Our setup is minimal and does not require exotic physics apart from the non-renormalisable term.

Acknowledgements $\mathrm{KD}$ is supported (in part) by the Lancaster-Manchester-Sheffield Consortium for Fundamental Physics under STFC grant: ST/L000520/1. CO is supported by the FST of Lancaster University. AR is supported by the Estonian Research Council grants IUT23-6, PUT1026 and by the ERDF Centre of Excellence project TK133.

[1] P. A. R. Ade et al. [BICEP2 and Keck Array Collaborations], Phys. Rev. Lett. 116 (2016) 031302.

[2] K. Dimopoulos and C. Owen, Phys. Rev. D 94 (2016) no.6, 063518

[3] R. Allahverdi, A. Kusenko and A. Mazumdar, JCAP 0707 (2007) 018.

[4] J. C. Bueno Sanchez, K. Dimopoulos and D. H. Lyth, JCAP 0701 (2007) 015.

[5] R. Allahverdi, K. Enqvist, J. Garcia-Bellido and A. Mazumdar, Phys. Rev. Lett. 97 (2006) 191304; D. H. Lyth, JCAP 0704 (2007) 006; R. Allahverdi, K. Enqvist, J. GarciaBellido, A. Jokinen and A. Mazumdar, JCAP 0706 (2007) 019; K. Enqvist, L. Mether and S. Nurmi, JCAP 0711 (2007) 014; R. Allahverdi, A. Ferrantelli, J. Garcia-Bellido and A. Mazumdar, Phys. Rev. D 83 (2011) 123507.

[6] N. Itzhaki and E. D. Kovetz, JHEP 0710 (2007) 054; M. Badziak and M. Olechowski, JCAP 0902 (2009) 010; K. Enqvist, A. Mazumdar and P. Stephens, JCAP 1006 (2010) 020; S. Hotchkiss, A. Mazumdar and S. Nadathur, JCAP 1106 (2011) 002; N. Okada and D. Raut, Phys. Rev. D 95 (2017) no.3, 035035.

[7] Y. Hamada, H. Kawai, K. Y. Oda and S. C. Park, Phys. Rev. Lett. 112 (2014) no.24, 241301; F. Bezrukov and M. Shaposhnikov, Phys. Lett. B 734 (2014) 249; G. Ballesteros and C. Tamarit, JHEP 1602 (2016) 153; N. Okada, S. Okada and D. Raut, Phys. Rev. D 95 (2017) no.5, 055030 .

[8] J. Khoury, B. A. Ovrut, P. J. Steinhardt and N. Turok, Phys. Rev. D 64 (2001) 123522. 
[9] R. H. Brandenberger, A. Nayeri, S. P. Patil and C. Vafa, Int. J. Mod. Phys. A 22 (2007) 3621.

[10] K. Kannike, G. Hütsi, L. Pizza, A. Racioppi, M. Raidal, A. Salvio and A. Strumia, JHEP 1505 (2015) 065; M. Rinaldi, L. Vanzo, S. Zerbini and G. Venturi, Phys. Rev. D 93 (2016) 024040; A. Farzinnia and S. Kouwn, Phys. Rev. D 93 (2016) no.6, 063528; L. Marzola, A. Racioppi, M. Raidal, F. R. Urban and H. Veermäe, JHEP 1603 (2016) 190; L. Marzola and A. Racioppi, JCAP 1610 (2016) no.10, 010; M. Artymowski and A. Racioppi, JCAP 1704 (2017) no.04, 007 .
[11] S. R. Coleman and E. J. Weinberg, Phys. Rev. D 7 (1973) 1888.

[12] M. S. Turner, Phys. Rev. D 28 (1983) 1243.

[13] W. H. Kinney, Phys. Rev. D 72 (2005) 023515; J. Martin, H. Motohashi and T. Suyama, Phys. Rev. D 87 (2013) no.2, 023514; M. H. Namjoo, H. Firouzjahi and M. Sasaki, Europhys. Lett. 101 (2013) 39001; A. E. Romano, S. Mooij and M. Sasaki, Phys. Lett. B 761 (2016) 119; C. Germani and T. Prokopec, arXiv:1706.04226 [astro-ph.CO].

[14] K. Dimopoulos, Phys. Lett. B 775 (2017) 262

[15] M. S. Turner, Phys. Rev. D 28 (1983) 1243.

[16] Y. Shtanov, J. H. Traschen and R. H. Brandenberger, Phys. Rev. D 51 (1995) 5438 [hep-ph/9407247]. 